\title{
Evaluación de la mortalidad neonatal en la Ciudad Autónoma de Buenos Aires según la residencia de la población y la pertenencia al subsistema de salud

\author{
Evaluation of neonatal mortality in Buenos Aires City by place of \\ residence and use of a health system subsector
}

\author{
Dr. Javier Meritano ${ }^{a}$, Dra. Lorena Tsavoussian ${ }^{a}$, Dr. Raúl Címbaro Canella ${ }^{a}$ \\ Dr. Claudio Solana ${ }^{a}$
}

\section{RESUMEN}

La mortalidad neonatal es el componente más importante de la mortalidad infantil. Su análisis es complejo y no depende exclusivamente del sistema sanitario.

En la Ciudad Autónoma de Buenos Aires (CABA), entre 2000 y 2012, la mortalidad neonatal fue inferior a la media nacional, pero no registró cambios. Además, la diferencia es cada vez menor: en el año 2000, era un $46 \%$ inferior; en 2012, se redujo a un $21 \%$.

Objetivo: Evaluar la asociación entre el lugar de residencia de la madre, el subsector de salud al cual pertenece y la tasa de mortalidad en los menores de 28 días de vida en la CABA.

Métodos: Estudio poblacional, transversal, durante los años 2011 y 2012, sobre datos del Registro Civil y de la Dirección de Estadísticas y Censos de la CABA.

Resultados: Se registraron 164837 nacimientos. Laproporción denacimientosenestablecimientos públicos y privados permanece estable; este último subsector presenta el $57 \%$ de los nacimientos. Los recién nacidos presentan similar proporción de género, peso y edad gestacional promedio en ambos subsistemas.

Hubo mayor mortalidad neonatal para madres no residentes de la CABA (6,55\% vs. 5,42\%o; OR 1,21;IC 95\%:1,07-1,37; p 0,0039). Para madres residentes en la CABA, hubo mayor mortalidad neonatal en el subsistema de salud público $(7,8 \%$ o vs. $4,4 \%$; OR 1,77 ; IC $95 \%$ : 1,48-2,11; $\mathrm{p}<0,001$ ). Conclusión: La CABA, único caso en el país, tiene un componente de nacimientos y defunciones extrajurisdiccionales muy importante, fundamentalmente del conurbano bonaerense, que no se refleja en las estadísticas oficiales que solo toman en cuenta el lugar de residencia de los padres.

Palabras clave: mortalidad neonatal, sistemas de salud, regionalización, factores epidemiológicos.

http:/ /dx.doi.org/10.5546/aap.2016.405

Financiamiento:

Ninguno.

Conflicto de intereses:

Ninguno que declarar.

Recibido: 20-11-2015

Aceptado: 21-4-2016

\section{INTRODUCCIÓN}

La mortalidad neonatal es el componente más importante de la mortalidad infantil y su análisis es complejo y no depende exclusivamente del sistema sanitario.

Desde el año 1980, en Argentina, se observa una tendencia al descenso en la tasa de mortalidad infantil (TMI) con ligeras oscilaciones. En los últimos 31 años, el descenso en la TMI ha sido de un $64,8 \%$, desde $33,2 \%$ en 1980 a $11,7 \%$ en 2011 , con una tasa de mortalidad neonatal (TMN) de 7,6\%. ${ }^{1}$

La TMN comprende $2 / 3$ de la TMI y es proporcionalmente mayor cuanto más baja es la TMI, como se puede observar en países desarrollados o en poblaciones con buenas condiciones socioeconómicas y culturales.

Globalmente, existe acuerdo en que la reducción de la mortalidad infantil en las últimas décadas en los países desarrollados es producto de la mayor supervivencia de los prematuros extremos. ${ }^{2,3}$ Las redes neonatales permiten mejorar la supervivencia y potenciar las mejores prácticas médicas. El lugar en que se nace es importante en la supervivencia del recién nacido. ${ }^{4}$

Las circunstancias en que las personas nacen, crecen, viven, trabajan y envejecen, incluido el sistema de salud, constituyen los determinantes sociales de la salud, que son la causa de la mayor parte de las desigualdades sanitarias entre los países y dentro de cada país. ${ }^{5}$ Estos determinantes son objetos de políticas públicas y susceptibles de modificación mediante intervenciones efectivas. 
En el período de veintidós años, entre 1990 y el 2011, en la Ciudad Autónoma de Buenos Aires (CABA), se observa una tendencia decreciente de la TMI, que implica una reducción del $47 \%$ (de $16 \%$ a $8,5 \%$ ). Sin embargo, las TMI de cada comuna registran importantes disparidades. ${ }^{6,7}$

En la CABA, la TMN no ha mejorado según lo previsto. En el período de trece años, entre 2000 y 2012, a pesar de que siempre fue inferior que la media nacional, no registra ningún cambio y la diferencia es cada vez menor: mientras, en el año 2000 , era un $46 \%$ inferior, se redujo a un $21 \%$ en 2012, según datos oficiales (Tabla 1). ${ }^{1}$

Se han realizado numerosas descripciones sobre esta problemática, pero no se sabe si existen asociaciones al respecto. Esto sería importante para poder realizar una evaluación $\mathrm{y}$, posteriormente, una intervención para lograr una mayor eficacia y eficiencia en los sistemas de salud con respecto a este problema.
El objetivo de este trabajo es evaluar la existencia de una asociación entre el lugar de residencia de la madre, el subsector de salud al cual concurrió y la tasa de mortalidad en los menores de 28 días de vida en la CABA, en los años 2011 y 2012.

\section{MATERIALES Y MÉTODOS}

Estudio poblacional, transversal. Para los datos de natalidad, se utilizó como fuente el Registro Civil de la Ciudad de Buenos Aires y, para las defunciones, la Dirección de Estadísticas y Censos de esta.

\section{POBLACIÓN \\ Criterios de inclusión}

Todos los registros de niños menores de 28 días nacidos y fallecidos en la CABA pertenecientes a la CABA y la provincia de Buenos Aires. Población seleccionada en forma consecutiva en los años 2011 y 2012.

Tabla 1. Comparación de la mortalidad neonatal en Argentina y en la Ciudad Autónoma de Buenos Aires

\begin{tabular}{lccccc}
\hline AÑO & Nacidos vivos Argentina & Nacidos vivos CABA & TMN \%o Argentina & TMN \% CABA & Diferencia \\
\hline 2000 & 701878 & 43587 & 10,9 & 5,9 & $46 \%$ \\
2001 & 683495 & 42171 & 10,6 & 6,2 & $42 \%$ \\
2002 & 694684 & 40825 & 11,3 & 6,3 & $44 \%$ \\
2003 & 697952 & 42136 & 10,5 & 6,4 & $39 \%$ \\
2004 & 736261 & 44019 & 9,7 & 5,7 & $41 \%$ \\
2005 & 712220 & 43064 & 8,9 & 5,2 & $42 \%$ \\
2006 & 696451 & 43582 & 8,5 & 4,9 & $42 \%$ \\
2007 & 700792 & 42183 & 8,5 & 5 & $41 \%$ \\
2008 & 746460 & 45122 & 8,3 & 4,9 & $41 \%$ \\
2009 & 745336 & 43584 & 7,9 & 5,8 & $28 \%$ \\
2010 & 756176 & 44347 & 7,6 & 4,5 & $43 \%$ \\
2011 & 758042 & 45280 & 7,5 & 5,5 & $29 \%$ \\
2012 & 738318 & 43733 & $31 \%$ & 5,9 & $21 \%$ \\
\hline \multicolumn{7}{c}{ Descenso de la TMN 2000-2012 } & & $0 \%$ & \\
\hline
\end{tabular}

Fuente: Dirección de Estadística e Información en Salud. Ministerio de Salud de la Nación.

CABA: Ciudad Autónoma de Buenos Aires; TMN: tasa de mortalidad neonatal.

TABLA 2. Variables del estudio según categorías

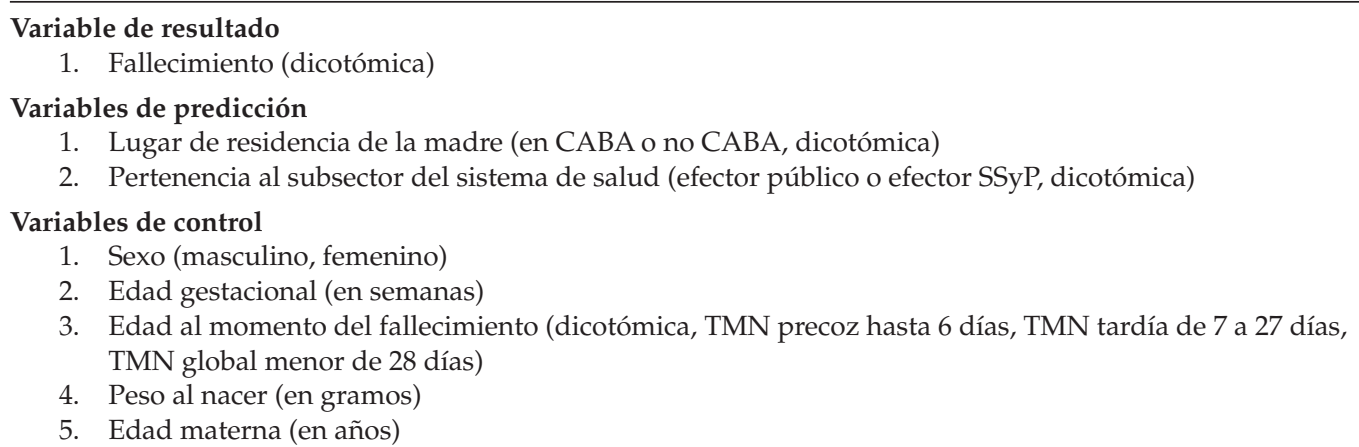

CABA: Ciudad Autónoma de Buenos Aires; SSyP: seguridad social/privado; TMN: tasa de mortalidad neonatal. 


\section{Criterios de exclusión}

Todos los registros que no estuviesen completos y que no fueran compatibles con los de nacimiento en peso, edad materna, sexo, fecha de nacimiento y código de establecimiento.

Para realizar la TMI, en este trabajo, se analizaron todos los recién nacidos en la CABA durante el período mencionado (numerador) y luego se construyó el denominador con todos los recién nacidos fallecidos que nacieron en la CABA. Esto se logró mediante el cruzamiento de las bases utilizando los registros de nacimiento en peso, edad materna, sexo, fecha de nacimiento y código de establecimiento.

\section{Variables}

Las variables se presentan en la Tabla 2 según categorías.

\section{Consideraciones éticas}

El estudio está asociado a una beca del Gobierno de la CABA y ha sido aprobado por el Comité de Ética en Investigación correspondiente.

\section{Análisis}

Los resultados se presentan en dos apartados diferentes: análisis de la natalidad y análisis de la mortalidad, en la que se presentan, además, datos sobre mortalidad en la subpoblación con peso al nacer entre 500 y 1499 gramos.

Se describió la incidencia de las variables en medias y se expresó en porcentajes con su intervalo de confianza del $95 \%$ o su desviación estándar, según correspondiera. Para evaluar la asociación entre las variables dicotómicas, se utilizó el test de chi cuadrado y se calcularon los odds ratio (OR) con su intervalo de confianza del 95\%.

Para un total de alrededor de 80000 recién nacidos por año en el territorio estudiado, esta población fue suficiente para identificar una diferencia de, al menos, un $0,04 \%$ en las variables de predicción para la variable de resultado con $95 \%$ de confianza y $80 \%$ de poder.

El análisis se realizó con el software estadístico EpiInfo ${ }^{\mathrm{TM}}$ versión 3.5.1 para Windows. Se consideró significativo un valor de $\mathrm{p}<0,05$.

TABLA 3. Características sociodemográficas de los niños nacidos en la Ciudad de Buenos Aires por residencia. Años 2011 y 2012

\begin{tabular}{|c|c|c|c|}
\hline \multirow{2}{*}{\multicolumn{2}{|c|}{ Total, n (\%) }} & CABA & \multirow{2}{*}{$\begin{array}{r}\text { NO CABA } \\
75592(45,9)\end{array}$} \\
\hline & & $89099(54,1)$ & \\
\hline a) & Sector público, n (\%) & $32374(34,8)$ & $34207(45,2)$ \\
\hline & Sexo masculino, $\mathrm{n}(\%)$ & $16434(50,8)$ & $17462(51)$ \\
\hline & Media de peso de nacimiento en gramos (DE) & $3359(580)$ & $3290(662)$ \\
\hline & Media de edad gestacional en semanas (DE) & $38,97(1,92)$ & $40,12(1,96)$ \\
\hline & Media de edad materna en años (DE) & $26,2(6,46)$ & $25,85(6,54)$ \\
\hline \multirow[t]{5}{*}{ b) } & Sector privado/seg. social, n (\%) & $56725(64,7)$ & $41385(54,6)$ \\
\hline & Sexo masculino, $\mathrm{n}(\%)$ & $29083(51,3)$ & $21098(51)$ \\
\hline & Media de peso de nacimiento en gramos (DE) & $3254(554)$ & $3225(626)$ \\
\hline & Media de edad gestacional en semanas (DE) & $38,51(1,9)$ & $38,95(1,9)$ \\
\hline & Media de edad materna en años (DE) & $31,79(5,59)$ & $30,72(6,01)$ \\
\hline
\end{tabular}

CABA: Ciudad Autónoma de Buenos Aires; DE: desvío estándar.

TABLA 4. Población materna agrupada por rango etario, residencia y pertenencia al subsistema de salud. Años 2011 y 2012. (Sin dato 2498)

\begin{tabular}{ccc}
\hline Residencia & \multicolumn{2}{c}{ CABA } \\
\hline Rango etario en años & Público & Seguridad social y privado \\
\hline $10-17, \mathrm{n}(\%)$ & $1773(5,8)$ & $486(0,8)$ \\
$18-45, \mathrm{n}(\%)$ & $28468(94)$ & $55611(98,7)$ \\
$46-60, \mathrm{n}(\%)$ & $17(0,05)$ & $199(0,3)$ \\
Residencia & & NO CABA \\
\hline Rango etario en años & Público & Seguridad social y privado \\
\hline $10-17, \mathrm{n}(\%)$ & $2347(6,9)$ & $736(1,8)$ \\
$18-45, \mathrm{n}(\%)$ & $31797(93)$ & $40522(98)$ \\
$46-60, \mathrm{n}(\%)$ & $26(0,07)$ & $108(0,2)$ \\
\hline
\end{tabular}

CABA: Ciudad Autónoma de Buenos Aires. 


\section{RESULTADOS}

\section{Análisis estadístico: natalidad}

Se registraron 164691 nacimientos durante los años 2011 y 2012. El número de nacimientos permanece estable en los dos años. La proporción de nacimientos en establecimientos públicos y de la seguridad social/privados (SSyP), también; este último subsector presenta mayor cantidad de nacimientos.

Los residentes de la CABA nacen en mayor proporción en el subsistema SSyP (64,7\%) (Tabla 3).

Los recién nacidos presentan similar proporción de género, peso, edad gestacional y edad materna promedio en los dos subsistemas y entre los residentes de la CABA y no CABA (Tabla 3).

El embarazo adolescente es una característica marcada en el subsector público y es más alto en los residentes no CABA. Si comparamos por grupos de edades, encontramos una diferencia estadísticamente significativa entre las chances de las madres adolescentes de la CABA de tener a sus hijos en hospitales públicos (OR 7,15; IC 95\%: 6,46-7,91; $\mathrm{p}<0,001)$. El resultado es similar en la población de no CABA, pero con menor fuerza de asociación (OR 4,07; IC 95\%: 3,74-4,43; p < 0,001).

A diferencia de lo anterior, la proporción de madres mayores de 17 años y mayores de 45 años es significativamente mayor en el subsector de salud SSyP de la CABA, con resultados similares en la población no CABA y menor fuerza de asociación (Tabla 4).

El análisis de las edades gestacionales no muestra diferencias; las cifras de recién nacidos prematuros extremos es similar en todas las poblaciones.
No hay diferencias entre las poblaciones en el análisis de nacimientos por peso. Los recién nacidos prematuros menores de 1500 gramos son similares en todos los subgrupos (Tabla 3).

\section{Análisis de la mortalidad}

Para disminuir los sesgos de los fallecidos no residentes de la CABA, se realizó el entrecruzamiento de las bases de mortalidad y natalidad. De los 693 fallecidos en los años 2011 y 2012, se confirmaron 491 (71\%) que nacieron y fallecieron en la CABA y no residían allí.

La TMI de la CABA es una de las más bajas del país, y la TMN es su mayor componente. En los residentes de la CABA, el subsector público duplica la mortalidad del subsector SSyP (7,8\%o vs. $4,4 \%$ o).

La TMN de los residentes no CABA es significativamente mayor que los residentes de la CABA (6,55\% vs. $5,42 \%$; OR 1,21; IC 95\%: $1,07-$ 1,37 ; p 0,003); a su vez, los residentes no CABA presentan mayor mortalidad en el subsector público (Tabla 5).

En los residentes de la CABA, las diferentes categorías de TMN son mayores y estadísticamente significativas en establecimientos públicos con respecto a los SSyP. Para la TMN precoz, se ha estimado un OR 1,72; IC 95\%: 1,42,$12 ; \mathrm{p}<0,001$. Para la TMN tardía, OR 1,92; IC 95\%: 1,34-2,73; $\mathrm{p}<0,001$. Y, para la TMN global, OR 1,77; IC 95\%: 1,48-2,11; p <0,001.

En los residentes no CABA, también hallamos las mismas diferencias, pero con diferente fuerza para la TMN precoz: OR 1,25; IC 95\%: 1,04-1,49; $p<0,05$. En este caso, con respecto a la TMN tardía, no hallamos diferencias significativas:

TABLA 5. Tasas de mortalidad neonatal y muertes agrupadas por categorías, residencia y subsistema de salud. Años 2011 y 2012

\begin{tabular}{|c|c|c|c|c|}
\hline Residencia & CABA & No CABA & OR (IC 95\%) & $p$ \\
\hline Tasa de mortalidad neonatal precoz, \%o (n) & $4(357)$ & $4,38(332)$ & $1,09(0,94-1,27)$ & 0,23 \\
\hline Tasa de mortalidad neonatal tardía, \%o (n) & $1,42(126)$ & $2,17(164)$ & $1,53(1,21-1,93)$ & $<0,001$ \\
\hline Tasa de mortalidad neonatal, \% (n) & $5,42(483)$ & $6,55(496)$ & $1,21(1,07-1,37)$ & 0,003 \\
\hline $\begin{array}{l}\text { Residencia en la CABA } \\
\text { Subsistema de salud }\end{array}$ & Público & SSyP & OR (IC 95\%) & $p$ \\
\hline Tasa de mortalidad neonatal precoz, \%о (n) & $5,6(171)$ & $3,3(186)$ & $1,72(1,4-2,12)$ & $<0,001$ \\
\hline Tasa de mortalidad neonatal tardía, \% (n) & $2,1(64)$ & $1,1(62)$ & $1,92(1,34-2,73)$ & $<0,001$ \\
\hline Tasa de mortalidad neonatal, \%o (n) & $7,8(235)$ & $4,4(248)$ & $1,77(1,48-2,11)$ & $<0,001$ \\
\hline $\begin{array}{l}\text { Residencia no CABA } \\
\text { Subsistema de salud }\end{array}$ & Público & SSyP & OR (IC 95\%) & $p$ \\
\hline Tasa de mortalidad neonatal precoz, \%о (n) & $5,09(174)$ & $3,81(158)$ & $1,33(1,08-1,66)$ & 0,0086 \\
\hline Tasa de mortalidad neonatal tardía, \%o (n) & $2,03(69)$ & $2,3(95)$ & $0,87(0,64-1,2)$ & 0,4175 \\
\hline Tasa de mortalidad neonatal, \%o (n) & $7,1(243)$ & $6,11(253)$ & $1,16(0,97-1,39)$ & 0,09 \\
\hline
\end{tabular}

CABA: Ciudad Autónoma de Buenos Aires; OR: odds ratio; IC: intervalo de confianza; SSyP: seguridad social/privado. 
OR 0,87; IC 95\%: 0,64-1,2; p 0,86. Y, para la TMN global, OR 1,16; IC 95\%: 0,97-1,39; p 0,09.

En el análisis de la mortalidad neonatal en la población entre 500 y 1499 gramos, en los residentes no CABA, la mortalidad es significativamente mayor (OR 2,09; IC 95\%: $1,61-2,71 ; \mathrm{p}<0,001)$. En los residentes de la CABA, el subsistema público presenta una mayor mortalidad que el SSyP (OR 1,61; IC 95\%: 1,06-2,47; $<<0,03)$. En los residentes no CABA, también hallamos la misma diferencia (OR 1,39; IC 95\%: 1,01-1,93; p 0,0441) (Tabla 6).

\section{DISCUSIÓN}

La primera conclusión a la que podemos arribar es que la CABA, único caso en el país, tiene un componente de nacimientos y defunciones extrajurisdiccionales muy importante, fundamentalmente del conurbano bonaerense, que no se refleja en las estadísticas oficiales que solo toman en cuenta el lugar de residencia de los padres. Esto refleja la impostergable necesidad de oficializar y articular las medidas para integrar en una sola región sanitaria, tanto asistencial como estadísticamente, la CABA con los primeros cordones poblacionales de la provincia de Buenos Aires. De lo contrario, por ejemplo, en lo que hace a estadísticas y resultados neonatales, solo estamos observando la mitad de la realidad.

La principal fortaleza de este estudio radica en la confiabilidad de los registros. La potencial debilidad que se le puede atribuir es que determinamos los fallecidos que realmente nacieron en la CABA y sus padres no tenían residencia en la CABA con el entrecruzamiento de las bases de natalidad y mortalidad (utilizando los registros de nacimiento en peso, edad materna, sexo, fecha de nacimiento y código de establecimiento).
La comparación de las cifras es llamativamente diferente según el lugar de residencia. Mientras los residentes en la CABA presentan unas de las TMN más bajas del país $(5,57 \%$ ), los no residentes atendidos por el mismo sistema sanitario presentan cifras más altas que la media nacional $\left(10,3 \%\right.$ vs. $7,59 \%$ o). ${ }^{8}$

Para el abordaje de esta problemática, es imprescindible reconocer que el sistema de salud argentino se caracteriza por su segmentación y fragmentación (regulación y territorio). Esto genera importantes barreras para el cumplimiento del derecho a la salud. ${ }^{9}$

Otro aspecto importante es que la cobertura de salud de la población se distribuye por niveles de ingreso. Un estudio nacional registra que la mayor dependencia de los servicios ofrecidos por el sector público aumenta a medida que disminuye el nivel de ingresos per cápita de las familias. ${ }^{9}$

Si evaluamos territorialmente, la región bonaerense presenta un 10\% más de fallecimientos infantiles entre municipios de peor y mejor situación, mientras que, en la CABA, este riesgo aumenta a un $150 \%$ entre las distintas comunas. ${ }^{10}$

Con respecto al modelo de atención, se ha copiado un modelo curativo, en el cual, por un lado, encontramos un incremento desmedido de equipamiento neonatal y nuevas técnicas y, por otro, servicios que tienen una elevada prevalencia de infecciones, poca enfermería entrenada, profesionales que no concurren a la institución, carecen de guías de trabajo o normas y no han completado el entrenamiento en una técnica cuando incorporan otra novedad. ${ }^{11}$

El número de controles prenatales podría brindar información sobre el cuidado del embarazo, el acceso precoz al sistema y las

TABLA 6. Mortalidad neonatal en población entre 500 y 1499 gramos por residencia y subsector del sistema de salud. Años 2011 y 2012

\begin{tabular}{ccccc}
\hline Residencia & CABA & No CABA & OR (IC 95\%) & $p$ \\
\hline Mortalidad neonatal, \% (n) & $10,23(103)$ & $19,28(182)$ & $2,09(1,61-2,71)$ & $<0,001$ \\
\hline $\begin{array}{c}\text { CABA } \\
\text { Subsistema de salud }\end{array}$ & Público & SSyP & OR (IC 95\%) & $p$ \\
\hline $\begin{array}{c}\text { Mortalidad neonatal, \% (n) } \\
\text { No CABA }\end{array}$ & $20(47)$ & $12(56)$ & $1,61(1,06-2,47)$ & $<0,03$ \\
Subsistema de salud & Público & SSyP & OR (IC 95\%) & $p$ \\
\hline Mortalidad neonatal, \% (n) & 22,05 (97) & $16,87(85)$ & $1,39(1,01-1,93)$ & 0,0441 \\
\hline
\end{tabular}

CABA: Ciudad Autónoma de Buenos Aires; OR: odds ratio; IC: intervalo de confianza; SSyP: seguridad social/privado. 
posibilidades de implementar medidas preventivas o de cuidados. En 2011, un 51,1\% de las embarazadas en el conurbano y un $57,8 \%$ en la CABA presentaron controles prenatales adecuados, ${ }^{12}$ muchas de las cuales no reunían los requisitos aceptables en cuanto a su precocidad, distribución, integralidad y calidad. Solo el 30\% de los controles se inicia precozmente durante el primer trimestre.

Aún es deficiente la articulación entre las actividades de promoción de la salud desarrolladas en las comunidades y los servicios de salud del primer nivel de atención, y de estos con las maternidades que concentran la atención de los partos y sus recién nacidos. Esta situación se ve agravada por la condición de pobreza, que contribuye a magnificar el impacto negativo de la falta de cuidado para preservar en la mujer su plena capacidad de salud reproductiva y el buen pronóstico del embarazo y de su hijo recién nacido. ${ }^{13}$

Alrededor de 70000 adolescentes en países en desarrollo mueren por año por causas relacionadas con el embarazo y el parto. Las adolescentes que quedan embarazadas tienden a ser de hogares de ingresos más bajos y a tener una nutrición deficiente. ${ }^{14-16}$ En nuestro estudio, el porcentaje calculado de menores de 18 años que dieron a luz en el subsector público fue 5 veces más elevado que en el subsector SSyP.

Lo que tienen en común es que las niñas que son pobres y analfabetas o tienen poca educación son más proclives a quedar embarazadas que las más ricas, urbanas y educadas. ${ }^{14} \mathrm{La}$ mortinatalidad, la morbilidad y la muerte del recién nacido son un 50\% más altas entre hijos de madres adolescentes que entre hijos de madres de entre 20 y 29 años de edad. ${ }^{17-19}$

En el análisis de la población entre 500 y 1499 gramos, la discusión está centrada en la proporción de enfermeras y el grado de complejidad de la atención. Se ha demostrado que la sobrevida se asocia con el número de enfermeras especializadas. ${ }^{20} \mathrm{El}$ incremento por encima de 1,2 enfermeras/pacientes disminuye la probabilidad de mortalidad en un $42 \%{ }^{20}$ Callaghan demostró que la chance de fallecer disminuyó un $82 \%$ cuando la razón de enfermera/paciente era superior a 1,7. ${ }^{3}$ Estudios realizados en nuestros contextos marcan también estas diferencias. $^{21}$

La inadecuada regionalización de la atención perinatal en el área metropolitana es otro aspecto muy importante que resulta del análisis. Los programas regionalizados se diseñaron con el fin de organizar los servicios para que las mujeres y niños de alto riesgo tuvieran su atención en hospitales provistos con la experiencia y la tecnología requeridas para asegurarles cuidados óptimos. Las maternidades fueron clasificadas en tres niveles de atención, se organizaron sistemas de transporte y se construyeron vínculos entre las estructuras de salud con el fin de mantener la capacitación en los niveles de menor complejidad que derivaban los casos de alto riesgo. Se han publicado experiencias realizadas en diferentes países, en las que fueron evaluados los resultados de los sistemas perinatales regionalizados sobre un indicador: la mortalidad neonatal, en especial, en recién nacidos de menos de 1500 gramos al nacer. En distintos contextos y sistemas de salud, la regionalización parece actuar positivamente en los indicadores materno-infantiles. Experiencias en Canadá, Portugal, Chile, Reino Unido avalan esta política como organización de la atención. ${ }^{22}$

Finalmente, predominan en el hospital público el embarazo adolescente, menos años de escolarización, la falta de controles, el menor uso de corticoides, la presencia de diabetes gestacional (relacionada con la malnutrición) y la corioamnionitis. Este mayor riesgo podría estar relacionado con la pobreza, la deficiente nutrición, el hábito de fumar, el alcoholismo y con la mala salud previa al embarazo. ${ }^{23}$

Se debe ampliar la investigación sobre la mortalidad neonatal, el lugar de residencia, las condiciones de vida, el acceso a la salud y los efectores de salud, que impactan directamente sobre las posibilidades de vida de los recién nacidos.

Esta discusión lleva a relevar el valor que nuestra sociedad otorga a la salud al plantear su centralidad para el desarrollo pleno de oportunidades por los individuos. La salud y la equidad en salud deben transformarse en objetivos colectivos que involucran a todos los sectores del Gobierno y de la sociedad civil y no solo al sector salud, menos aún restringirse al sistema de atención de salud exclusivamente.

El desafío es, entonces, empezar a conocer las particularidades de nuestras inequidades en salud y sus determinantes, que se comportan de manera distinta en los distintos sectores de nuestra población.

\section{CONCLUSIÓN}

La CABA, único caso en el país, tiene un componente de nacimientos y defunciones 
extrajurisdiccionales muy importante, fundamentalmente del conurbano bonaerense, que no se refleja en las estadísticas oficiales que solo toman en cuenta el lugar de residencia de los padres.

Se deben realizar estudios que profundicen el conocimiento de los determinantes sociales asociados.

\section{REFERENCIAS}

1. Argentina. Ministerio de Salud de la Nación. Dirección de Estadística e Información en Salud. Estadísticas vitales. Información básica año 2011. Buenos Aires: Ministerio de Salud de la Nación; 2012. [Acceso: 1 de septiembre de 2014]. Disponibleen:http://www.bvs.org.ar/pdf/anuario11.pdf.

2. Ramos Ramos A, Martínez Ramírez A, Morales Fernández A, Valdez López RM. La prematurez y sus repercusiones en el crecimiento y desarrollo del niño, en la zona metropolitana de Guadalajara, Jalisco, México. Cad Saude Publica 1998;14(2):313-8.

3. Callaghan LA, Cartwright DW, O'Rourke P, Davies MW. Infant to staff ratios and risk of mortality in very low birthweight infants. Arch Dis Child Fetal Neonatal Ed 2003;88(2):F94-7.

4. Hübner ME, Nazer J, Juárez de León G. Estrategias para mejorar la sobrevida del prematuro extremo. Rev Chil Pediatr 2009;80(6):551-9.

5. Organización Mundial de la Salud. Determinantes sociales de la salud. Subsanar las desigualdades en una generación: alcanzar la equidad sanitaria actuando sobre los determinantes sociales de la salud. Informe. Ginebra: OMS; 2009. [Acceso: 1 de septiembre de 2014]. Disponibleen:http://www.who.int/social_determinants/ thecommission/finalreport/es/.

6. Vettore MV, Gama SG, Lamarca Gde A, Schilithz AO, et al. Housing conditions as a social determinant of low birthweight and preterm low birthweight. Rev Saude Publica 2010;44(6):1021-31.

7. Gobierno de la Ciudad de Buenos Aires. Dirección General deEstadísticas y Censos. La mortalidad infantilen la Ciudad de Buenos Aires 2000-2011. Buenos Aires: GCBA; 2012. [Acceso: 1 de septiembre de 2014]. Disponible en: http:/ / www.estadisticaciudad.gob.ar/eyc/?p=38773.

8. Sociedad Argentina dePediatría, UNICEF Argentina. Salud materno-infanto-juvenil en cifras 2013. Buenos Aires: SAP; 2013. [Acceso: 21 de abril de 2016]. Disponible en: http:/ / www.unicef.org/argentina/spanish/salud_SapUnicef_ cifras2013.pdf.

9. Organización Panamericana de la Salud. Programa Naciones Unidas para el Desarrollo. El sistema de salud argentino y su trayectoria de largo plazo: logros alcanzados y desafíos futuros. Buenos Aires: PNUD; 2011. [Acceso: 21 de abril de 2016]. Disponible en: http://www.undp. org/content/argentina/es/home/library/human_ development/aportes-para-el-desarrollo-humano-enargentina-2011--el-sistema-.html.

10. Comes Y, Fures NO. Estudio comparativo de las desigualdades sociales en la mortalidad infantil en el área metropolitana de Buenos Aires - Año 2011. Epidemiología y Salud 2012;1(1):6-10.

11. De Sarasqueta P. ¿Cuál es el modelo? Rev Hosp Matern Infant Ramon Sardá 2001;20(2):50-1.

12. Argentina. Ministerio deSalud dela Nación. Primer informe nacional de relevamiento epidemiológico SIP-Gestión Buenos Aires: Organización Panamericana dela Salud;2013. [Acceso: 1 de septiembre de 2015]. Disponible en: http:/ / publicaciones.ops.org.ar/publicaciones/publicaciones $\% 20$ virtuales/SIP-G_Anexos/pdfPublicacion/SIP-G.pdf.

13. Argentina. Ministerio de Salud de la Nación. Guía de Seguimiento del Recién Nacido de Riesgo: Unidad Coordinadora de Programas Materno Infantiles Comisión y Nutricionales. Buenos Aires: Ministerio de Salud de la Nación; 2001.

14. Fondo de Población de las Naciones Unidas. Maternidad en la niñez: enfrentar el reto del embarazo en adolescentes. New York: UNFPA; 2013. [Acceso: 21 de abril de 2016]. Disponibleen:http://www.unfpa.org.mx/publicaciones/ SP-SWOP2013.pdf.

15. Gogna M. Embarazo y maternidad en la adolescencia. Estereotipos, evidencias y propuestas para políticas públicas. Buenos Aires: CEDES-UNICEF; 2005.

16. World Health Organization. Global and regional estimates of violence against women: prevalence and health effects of intimate partner violence and non-partner sexual violence. Geneva: WHO; 2013.

17. Olausson PO, Cnattingius S, Haglund B. Teenage pregnancies and risk of late fetal death and infant mortality. Br J Obstet Gynaecol 1999;106(2):116-21.

18. Draper ES, Manktelow B, Field DJ, James D. Prediction of survival for preterm births by weight and gestational age: retrospective population based study. BMJ 1999;319(7217):1093-7.

19. Bolzán A, Norry M, Borguetti M, Velez M, et al. La embarazada adolescente como grupo de riesgo obstétrico de dos distritos de la provincia de Buenos Aires, 1996-1997. Obstet Ginecol Latinoam 1998;56(2):80-6.

20. Hamilton KE, Redshaw ME, Tarnow-Mordi W. Nurse staffing in relation to risk-adjusted mortality in neonatal care. Arch Dis Child Fetal Neonatal Ed 2007;92(2):99-103.

21. Grandi C, González A, Meritano J, Grupo Colaborativo Neocosur. Riesgo de morbimortalidad neonatal de recién nacidos menores de 1500 gramos asociado al volumen de pacientes, personal médico y de enfermería: una investigación multicéntrica latinoamericana. Arch Argent Pediatr 2010;108(6):499-510.

22. Argentina.MinisteriodeSalud delaNación. Regionalización de la atención perinatal. Mayo 2010. Buenos Aires: Ministerio de Salud de la Nación; 2011.

23. Alazraqui M,Spinelli H. Desigualdades en salud en el nivel local-municipal. Buenos Aires: Universidad Nacional de Lanús; 2008:153-62. 